\title{
Vitamin D in Health and Disease
}

\author{
Dr. Harmanjot Kaur ${ }^{1 *}$, Dr. Roopjot Kochar ${ }^{2}$ \\ ${ }^{1}$ Consultant Nutritionist, SGS Ayurveda and Nutrition, Khanna, Punjab, India \\ ${ }^{2}$ Director, SGS Ayurveda and Nutrition, Khanna, Punjab, India
}

\author{
*Corresponding Author: Dr. Harmanjot Kaur, Consultant Nutritionist, SGS Ayurveda and Nutrition, \\ Khanna, Punjab, India, Email: harmankochar@yahoo.co.in
}

\begin{abstract}
Vitamin D is one of the most essential micronutrient required by our body. It is aptly called the sunshine vitamin, as it is majorly synthesized by the ultraviolet B (UVB) light obtained from the sun, which is then converted into its active form in the liver and kidneys. Studies found that vitamin D plays a vital part not only in bone health, but also in non-skeletal functions such as regulation of gene expression and enhancing the immune system. However, the key issue is inadequate amount of vitamin D in our body, due to low dietary intake and limited sunlight exposure. Vitamin D deficiency poses a significant public health problem due to its association with osteoporosis, osteomalacia, fractures, risk of cancer, diabetes, heart disease and other chronic illnesses. It is necessary for all individuals of any age group to consume the recommended amounts of vitamin D. some measures to replenish its deficiency is sunlight exposure, food fortification and routine supplementation. This review article emphasizes the role of vitamin $D$ in the body, prevalence of vitamin $D$ deficiency, potential implications along with strategies for treatment and prevention of its deficiency.
\end{abstract}

Keywords: Vitamin D, sunlight, bone, deficiency, supplementation, fortification.

\section{INTRODUCTION}

Vitamin D has been traditionally known as antiricketic factor or sunshine vitamin. Vitamin $\mathrm{D}$ is not really a vitamin at all. It is a steroid which, in its active form, has a hormone activity. By definition, Vitamin D is a hormone because it is made in one organ (skin) and transported by body fluid (blood) to act or activate other body parts. [1] Ancient civilizations worshipped the sun for its life-giving power, and it is well known how plants utilize this energy to make food, Vitamin D owes its production in humans to the sun too. Also called as the sunshine vitamin, it is the only essential vitamin that is also obtained from a non-food source- from exposure to sunlight [2].

\section{Conversion OF PRE-Vitamin D INTO VITAMIN D3}

Usually, 50\%-90\% of Vitamin D is produced by sun exposure of skin, and the remainder comes from the diet. The sun rays provide the ultraviolet B (UVB) energy, which causes photolysis of 7- dehydrocholesterol in the skin to create a precursor of vitamin D i.e. Previtamin D (pre-cholecalciferol). The liver and kidneys then convert this into the active form called $\quad 1 \alpha, \quad$ 25-dihydroxyvitamin $\quad \mathrm{D}$ (cholecalciferol). 1 $\alpha, 25$-dihydroxyvitamin D (Calcitriol) by binding to the Vitamin D Receptor (VDR), regulates the expression of several genes contributing to several skeletal and biological functions [3]. The ability to form precholecalciferol is affected by various factors such as latitude, season and diurnal variations, zenith angle and time of day. Atmospheric pollution weakens solar radiation. Dress code, skin pigmentation, and application of sun protection factor of 15 reduce the UVB penetration into epidermis by more than $95 \%$, thereby limiting the production of Vitamin D3 [4].

\section{GROUPS AT RISK OF VITAMIN D INADEQUACY}

\subsection{Breastfed Infants}

Vitamin D requirements cannot ordinarily be met by human milk alone, which provides $<25$ $\mathrm{IU} / \mathrm{L}$ to $78 \mathrm{IU} / \mathrm{L}$. Vitamin D content of human milk is related to the mother's vitamin D status; therefore mothers who supplement with high doses of vitamin D may have high levels of vitamin D in their milk. American Association of Pediatricians (AAP) recommends that 
exclusively and partially breastfed infants must be supplemented with $400 \mathrm{IU}$ of vitamin D per day, the recommended daily allowance for this nutrient during infancy [5].

\subsection{Older Adults}

Older adults are at high risk of developing vitamin D insufficiency because of aging. Their skin cannot synthesize vitamin D as efficiently, they are likely to spend more time indoors, and they may have inadequate intakes of the vitamin [5].

\subsection{People with Limited Sun Exposure}

Homebound individuals, women who wear long robes and head coverings for religious reasons and people with occupations that limit sun exposure are unlikely to obtain adequate vitamin $\mathrm{D}$ from sunlight. The significance of the role that sunscreen may play in reducing vitamin D synthesis is still unclear. Intake of RDA levels of vitamin D from foods and/or supplements will provide adequate amounts of this nutrient to these individuals.

\subsection{People with Dark Complexion}

Larger amounts of the pigment melanin in the epidermal layer result in darker skin and reduce the skin's ability to produce vitamin D from sunlight. It is not sure that lower levels of $25(\mathrm{OH}) \mathrm{D}$ for persons with dark skin have significant health consequences. Intake of RDA levels of vitamin $D$ from foods and/or supplements will provide adequate amounts of this nutrient to these individuals [2].

\subsection{People with Fat Malabsorption}

Vitamin D is fat soluble, therefore it requires some dietary fat in the gut for absorption. Individuals with reduced ability to absorb dietary fat might require vitamin D supplements. Fat malabsorption is associated with a variety of medical conditions including some forms of liver disease, cystic fibrosis, and Crohn's disease.

\subsection{People Who Are Obese or Who Have Undergone Gastric Bypass Surgery}

A BMI value of $\geq 30$ is associated with lower serum $25(\mathrm{OH})$ D levels compared with non obese individuals. Obese people may need larger than usual intakes of vitamin $\mathrm{D}$ to achieve $25(\mathrm{OH}) \mathrm{D}$ levels comparable to those of normal weight. Greater amounts of subcutaneous fat sequester (captivate) more of the vitamin and alter its release into the circulation. Individuals who have undergone gastric bypass surgery may become vitamin $\mathrm{D}$ deficient over time without a sufficient intake of vitamin D from food or supplements; moreover part of the upper small intestine where vitamin $\mathrm{D}$ is absorbed is bypassed [5].

\section{Physiological Forms AND Food SOURCES FOR VITAMIN D}

Vitamin D, a fat-soluble vitamin, is stored in the body for a long time. Two primary dietary forms exist:

4.1.Vitamin D3 (Cholecalciferol): Found in egg yolk and fatty fish.

4.2.Vitamin D2 (ergocalciferol): Found in some plants, mushrooms and yeasts.

In an economically diverse country like India, dietary sources rich in vitamin D are not easily accessible or affordable for most Indians. Vegetables and grains are poor sources of vitamin D. Dietary sources of vitamin D include fatty fish (salmon, tuna, sardines, swordfish), cod liver oil, egg yolks, portabella mushrooms, beef liver, and fortified foods such as breakfast cereal, milk (dairy and nondairy), infant formula, cheese, and orange juice. Vitamin D supplements are available; however, awareness of their need is not very widespread in the Indian population. Probably a more viable option to combating vitamin D deficiency in India would be fortification of staple foods such as rice and beverages such as milk $[6,7]$.

\section{ROLE OF VITAMIN D IN OUR BODY}

Vitamin D is one of the key nutrients required to conduct various physiological functions of the body, such as:

\subsection{Absorption of Calcium}

Vitamin D, along with calcium, helps build bones and keep bones strong. 1.25Dihydroxyvitamin D3 is the most important biologically active controlling hormone, for intestinal calcium absorption. Severe deficiency of Vitamin D gives rise to rickets in children and osteomalacia in adults.

\subsection{Block The Release of Parathyroid Hormone}

The regulation of calcium homeostasis is also caused by parathyroid hormone and ionized calcium. This hormone reabsorbs bone tissue, which makes bone thin and brittle. Presence of Vitamin D blocks this hormone resulting in the restoration of bone health [8]. 


\subsection{Calcium and Phosphorus Homeostasis}

The Vitamin D functions in the intestine, bones and kidneys to stimulate the transport of calcium and phosphorus into the extracellular fluid compartment in the target organs [8].

\subsection{Boost Muscle Function}

Daily Vitamin D consumption has been shown to improve tests of muscle performance, reduce the risk of falling and potentially the impact on muscle fiber composition and morphology in Vitamin D deficient older adults [9].

\subsection{Regulation of Cell Differentiation and Growth}

Vitamin D is now being known for its potential antiproliferative, pro-differentiation and immunomodulatory roles. It can regulate cell differentiation and growth by binding to the VDF's that are found in most body cells [10].

\section{VITAMIN D DEFICIENCY IN INDIA}

Vitamin D deficiency is one of the world's most common nutritional deficiencies and yet it is the most under-diagnosed and under-treated out of all the global nutritional deficiencies. With a prevalence of 50-90 per cent in the general population, the deficiency of this vitamin has reached epidemic proportions across the Indian subcontinent. In India, extensively consumed food items are rarely fortified with Vitamin D, greater than 50-90 percent of Vitamin D originates from the skin's exposure to sunlight. Unfortunately, today's new age lifestyles do not facilitate adequate sun exposure, leaving Indians to miss out on the potential health benefits of adequate sunshine. It is quite likely that the deficiency of Vitamin D plays an important role in the high occurrence of osteoporosis, cardiovascular diseases, cancer, rickets, diabetes and infections such as tuberculosis in India [11].

\subsection{Vitamin D Deficiency and Associated Co- Morbidities}

Vitamin D deficiency is one of the most globally widespread nutritional deficiencies. Current research has documented that 25hydroxyvitamin D deficiency is correlated to the onset and progression of many chronic diseases. It is linked to a variety of co-morbid conditions such as hypertension, fibromyalgia, osteoporosis, depression, hypertension, obesity, chronic fatigue, syndrome, neurodegenerative diseases like Alzheimer's and diabetes.
6.2. Vitamin D Deficiency and Risk of Cardiovascular Diseases (CVD)

Vitamin D deficiency disturbs the epigenetic factors involved in the control of vasoconstriction and vasodilatation, the imbalance between which results in hypertension. Low levels of vitamin D result in the activation and release of certain proinflammatory cytokines that increase the risk of CVD. Vitamin D deficiency is associated with greater risk of cardiovascular diseases (e.g., stroke, sudden cardiac death) and their risk factors (e.g., hypertension, diabetes).

\subsection{Vitamin D Deficiency and Risk of Cancer}

A variety of epidemiologic studies have demonstrated that the levels of 25hydroxyvitamin D below $20 \mathrm{ng} / \mathrm{ml}$ are associated with a 30 to 50 per cent increased risk of incident colon, prostate and breast cancer, leading to higher rates of mortality due to these cancers. A 60-77 per cent decrease in the relative risk of cancer in post-menopausal women who increased their vitamin D intake by 1100 IU of vitamin D3 asserts the need to be sufficient in vitamin D [12].

\subsection{Vitamin D Deficiency and Risk of Diabetes}

Vitamin D plays a key role in improving pancreatic $\beta$-cell function, decrease insulin resistance, and improve systemic inflammation.25-hydroxyvitamin D levels have been negatively correlated with the prevalence of type 2 diabetes mellitus, islet $\beta$-cell function and insulin resistance and positively correlated with insulin sensitivity [13].

\subsection{Vitamin D Deficiency and Immune System Diseases}

Variety of immune cells such as the monocytes, macrophages, dendritic cells, T cells and B cells express VDR. Vitamin D plays a crucial role in regulating the immune responses by binding to VDR and modulates both activation and deactivation of the innate and adaptive responses. The ultimate results of this interaction include.

- Delayed progress of chronic inflammatory auto-immune diseases.

- Increased macrophages control over infections.

- Increased formation of memory B cells and B cell apoptosis. 


\subsection{Vitamin D Deficiency and Neuropsychiatric Disorders}

Vitamin D is involved in the development and functioning of brain cells. Study shows that 25hydroxyvitamin $\mathrm{D}$ deficiency has been associated with increased risk for neuropsychiatric disorders such as depression, Alzheimer's disease, epilepsy and neurocognitive decline [13].

\subsection{Vitamin D and Its Role in Pregnant and Lactating Women}

Vitamin D plays a vital role in maintaining the optimal health status of both the mother and the fetus. Current studies have shown a correlation between vitamin $\mathrm{D}$ deficiency and adverse pregnancy outcomes. Adequate nutritional vitamin D status during pregnancy is important for fetal skeletal development, tooth enamel formation and general fetal growth and development. There also is increasing evidence to suggest that vitamin D deficiency impacts on the immune function of the mother, the neonate and the infant through the first year of life.

\subsection{Effectiveness of Vitamin Supplementation during Pregnancy}

D

400 IU vitamin D/ day are inadequate in achieving sufficiency for the majority of pregnant women. To attain optimal production of vitamin D, 25-hydroxyvitamin D level of 40 $\mathrm{ng} / \mathrm{ml}(100 \mathrm{nmol} / \mathrm{l})$ or higher is required [14].

\subsection{Measures to Tackle Deficiency}

A common but erroneous belief is that adequate vitamin $\mathrm{D}$ can be obtained via diet alone. Barring fatty fish, the amount of vitamin D present in most foods is relatively low. There is a need to employ long-term strategies to address India's growing deficiency of Vitamin D [15]. These strategies include:

\subsubsection{Food Fortification}

All grades of milk can be fortified. Oil and milk products such as curd, yogurt, infant formulas, and butter can be fortified with Vitamin D. Widely consumed food items such as atta, maida, and rice flour can also be fortified. Vitamin D fortified food items should be made available to the public at minimal cost and be included in the public distribution system. In India, Vanaspati (dalda) is fortified with $200 \mathrm{IU}$ of Vitamin D per 100 g. Milk products of certain brand are also fortified with Vitamin D
6.9.2. Increased Screening for Vitamin D Levels to Diagnose Deficiency

Testing facilities for Vitamin D levels should be made affordable and accessible to those at high risk of clinical Vitamin D deficiency (pregnant women, children, elderly especially women), as mass screening is not feasible.

\subsubsection{Public education for increasing awareness}

Educational programs are a must to create awareness about Vitamin D deficiency as it is the most under diagnosed and undertreated nutritional disease. Both physicians and the public should be made aware of its implications. To develop, launch and sustain such a program, adequate investment in the form of time, money, and effort is required

\subsubsection{Use of Vitamin D supplementation}

Vitamin D supplements of good quality should be made available at PHC level for the population at risk, i.e., pregnant women, lactating women, children, and elderly.

\subsubsection{National health policies to support the above}

Government should support research groups to study and monitor the impact of supplementation programs and fortification strategies.

\section{VITAMIN D SUPPLEMENTATION}

To combat the high occurrence of Vitamin D deficiency in India, the Endocrine Society of India has recommended Vitamin D supplementation. These recommendations suggest varying daily / weekly Vitamin D2 or Vitamin D3 for six weeks for different age groups.

Table1. (Therapy Duration: 6 weeks) treatment regimens for Vitamin D deficiency [16]

\begin{tabular}{|l|l|l|}
\hline Age Group & Daily Dose (IU) & Maintenance (IU) \\
\hline$\leq 1$ month & 2000 & $400-1000$ \\
\hline $1-12$ months & 2000 & $400-1000$ \\
\hline ears & 5000 & $600-1000$ \\
\hline$>18$ years & 6000 & $1500-2000$ \\
\hline Special cases* & $6000-10000$ & $3000-6000$ \\
\hline
\end{tabular}

*Obese patients, patients with malabsorption syndromes, patients on medication which affect vitamin metabolism

\section{CONCLUSION}

Vitamin D is significant for our overall health. It is known to play a significant role in our body to regulate various functions such as calcium and phosphorus metabolism, immune system and gene regulation as well as improve muscle function. Research has shown that many people 
have lower than optimal Vitamin D levels. Hypovitaminosis of Vitamin D most commonly results from the inadequate intake. The recommended safe and tolerable upper limit for Vitamin D is $2000 \mathrm{IU} / \mathrm{d}$ for those aged one year and older.

\section{REFERENCES}

[1] Mostafa WZ, Hegazy RA. Vitamin D and the skin: Focus on a complex relationship: A review. J Adv Res. 6:793-804, (2015).

[2] Aparna P, Muthathal S, Nongkynrih B, Gupta SK. Vitamin D deficiency in India. J Family Med Prim Care. 7(2):324-330, (2018).

[3] Garg S, Dasgupta A, Maharana SP, Paul B, Bandyopadhyay L, Bhattacharya A. Sun exposure and Vitamin D in rural India: A cross sectional study. Indian J Public Health. 62:175181, (2018).

[4] Harinarayan CV, Holick MF, Prasad UV, Vani PS, Himabindu G. Vitamin D status and sun exposure in India. Dermatoendocrinol. 5:130141, (2013).

[5] Institute of Medicine (US) Committee to Review Dietary Reference Intakes for Vitamin D and Calcium; Ross AC, Taylor CL, Yaktine $\mathrm{AL}$, et al., editors. Dietary Reference Intakes for Calcium and Vitamin D. Washington (DC): National Academies Press (US); 2011.

[6] Compher CW, Badellino KO, Boullata JI. Vitamin D and the bariatric surgical patient: A review. Obes Surg. 18:220-224, (2008).

[7] Kim M. Pfotenhauer, DO Jay H. Shubrook, DO. Vitamin D Deficiency, Its Role in Health and Disease, and Current Supplementation
Recommendations. Am Osteopath Assoc. 117(5): 301-305, (2017).

[8] Veldurthy V, Wei R, Oz L, Dhawan P, Jeon YH, Christakos S. Vitamin D, calcium homeostasis and aging. Bone Res. 4:16041, (2016).

[9] Hossein-nezhad A, Spira A, Holick MF. Influence of vitamin D status and vitamin D3 supplementation on genome wide expression of white blood cells: a randomized double-blind clinical trial. PLoS One. 8(3), (2013).

[10] Almeida LF, Coimbra TM. Vitamin D Actions on Cell Differentiation, Proliferation and Inflammation. Int J Complement Alt Med. 6(5): 00201, (2017).

[11] Singh P. Treatment of Vitamin D Deficiency and Co morbidities: A Review. J Assoc Physicians India. 66(1):75-82, (2018).

[12] H Wang. Vitamin D and chronic diseases. Aging Dis. 8(3): 346-353, (2017).

[13] Tiwari P, Sharma N. Role of Vitamin D in Various Illnesses: A Review. J Pharma Care Health Sys. 4: 176, (2017).

[14] Wagner CL, Taylor SN, Johnson DD, Holis BW. The role of vitamin D in pregnancy and lactation: emerging concepts. Women's health (London, England). 8(3): 323-340, (2012).

[15] Kennel KA, Drake MT, Hurley DL. Vitamin D deficiency in adults: when to test and how to treat. Mayo Clinic Proceedings. 85(8): 752-757, (2010).

[16] Singh P. Treatment of Vitamin D Deficiency and Comorbities: A Review, J Assoc Phy Ind. 66: 75-82, (2018).

Citation: Harmanjot Kaur, Roopjot Kochar. Vitamin D in Health and Disease. ARC Journal of Nutrition and Growth. 2019; 5(2):6-10. DOI: dx.doi.org/ 10.20431/2455-2550.0502002.

Copyright: (c) 2019 Authors. This is an open-access article distributed under the terms of the Creative Commons Attribution License, which permits unrestricted use, distribution, and reproduction in any medium, provided the original author and source are credited. 